\title{
RESERVORIO ILEAL, RESULTADOS FUNCIONALES
}

ILEAL RESERVOIR, FUNCTIONAL RESULTS

RESERVATÓRIO ILEAL, RESULTADOS FUNCIONAIS

\section{ENRIQUE ORTIZ QUEVEDO, ANDRÉS CEDEÑo RUIZ, GABRIELA SUÁREZ ESPINOZA, MARÍA FLORES PINOS, JHON GÓMEZ TABARES, MALFY BENAVIDES PERDOMO}

Clínica Guayaquil; Guayaquil, Ecuador

\section{Resumen}

Objetivo: presentar la experiencia y resultados de corto plazo del Reservorio lleal y Anastomosis Reservorio ileo-anal (RIARA) en "J" luego de proctocolectomía en el manejo de Colitis Ulcerosa (CU) y Poliposis Adenomatosa Familiar (PAF). Métodos: revisión de los expedientes clínicos de una serie de casos manejados con RIARA evaluados entre 2014 y 2016. Resultados: se incluyeron 11 casos (8 hombres y 3 mujeres), de los cuales 8 tenían PAF y 3 CU. La edad media fue de 45 años. Se encontró absceso pélvico en tres casos y en uno, incontinencia anal a líquidos de manera temporal. Sólo 1 caso requirió re-intervención quirúrgica. La media de estancia hospitalaria fue de 11.9 días con un número de 5 evacuaciones en 24 horas a los tres meses. No hubo muertes en la serie. Conclusiones: los resultados de esta serie demuestran adecuada calidad de vida y buenos resultados funcionales, morbilidad aceptable y sin mortalidad. Es necesario el seguimiento a largo plazo para identificar complicaciones tardías.

PALABRAS CLAVE: colitis ulcerosa, pólipos, proctocolectomía.

Abstract

Objective: present the experience and short-term results of the lleal reservoir with ileo-anal anastomosis (CP-IAA) in "J" after proctocolectomy in the management of Ulcerative Colitis (UC) and Familial Adenomatous Polyposis (FAP). Methods: a review of clinical records managed with CP-IAA were evaluated in 2014 and 2016. Results: 11 cases ( 8 men and 3 women) were included, of which, 8 cases had FAP and 3 UC. The mean age was 45 years. A pelvic abscess was found in three cases and one anal fluid incontinence temporarily. Only 1 case required surgical re-intervention. The average hospital stay was 11.9 days with some 5 evacuations in 24 hours after three months. There were no deaths in the series. Conclusions: results of this series demonstrate the adequate quality of life and good functional results, acceptable morbidity, and no mortality. Long-term follow-up is necessary to identify late complications.

KEYWORDS: colitis ulcerative, polyps, proctocolectomy.

Objetivo: apresentar a experiência e os resultados a curto prazo do reservatório ileal e da anastomose do reservatório ileo - anal (RIARA) em "J" após proctocolectomia no tratamento da colite ulcerosa (UC) e polipose adenomatosa familiar (PAF). Métodos: revisão dos prontuários de uma série de casos tratados com RIARA avaliados entre 2014 e 2016. Resultados: foram incluídos 11 casos ( 8 homens e 3 mulheres), dos quais 8 com PAF e 3 com UC. A idade média foi de 45 anos. Abscesso pélvico foi encontrado em três casos e em uma incontinência anal fluida temporariamente. Apenas 1 caso requereu reoperação. 0 tempo médio de internação foi de 11,9 dias, com um número de 5 evacuações em 24 horas após três meses. Não houve mortes na série. Conclusões: os resultados desta série demonstram qualidade de vida adequada e bons resultados funcionais, morbidade aceitável e sem mortalidade. 0 acompanhamento a longo prazo é necessário para identificar complicações tardias. Palavras-chave: colite ulcerosa, pólipos, proctocolectomia

PALAVRAS-CHAVE: colite ulcerative, pólipos, proctocolectomia. 


\section{INTRODUCCIÓN}

El reservorio ileal con anastomosis ileo-anal (RIARA) es el procedimiento de elección en la reconstrucción del tránsito intestinal luego de una coloproctectomía por Poliposis Adenomatosa Familiar (PAF), Enfermedad De Crohn (EC) o Colitis Ulcerosa (CU). ${ }^{1}$ En 1978 Parks y Nicholls inician la era de la anastomosis ileo-anal con reservorio ileal en "S". ${ }^{2}$ Luego Utsunomiya y Beart en 1980 simplifican esta técnica creando un reservorio en "J" dado su fácil realización, ${ }^{3}$ mejores resultados funcionales, menor trauma esfinteriano y ausencia de necesidad de cateterización. Otras variantes han sido publicadas como el pouch en "H" por Fonkalsrud en 1985 y en "W" por Nicholls en 1987. ${ }^{4}$ Posteriormente en 1992 este procedimiento fue realizado por vía laparoscópica por Peters y colaboradores. ${ }^{5}$

Si bien la proctocolectomía permite la curación de portadores de CU y PAF, el procedimiento quirúrgico no está exento de riesgos y puede comprometer la función intestinal, donde la frecuencia y continencia son los parámetros más expuestos. ${ }^{6}$ En comparación con las otras alternativas terapéuticas, la principal ventaja del RIARA es la eliminación de la mucosa rectal enferma con conservación de la función esfinteriana. ${ }^{7}$ Esta operación ha demostrado ser reproducible, segura y efectiva en la mayoría de los casos, aunque se asocia a complicaciones postoperatorias inmediatas no menores del 27 al 50\%.8

Los resultados funcionales de esta técnica muestran sistemáticamente una significativa mejoría en la calidad de vida en el 80 a $90 \%$ de los pacientes; aunque no está exenta de complicaciones que aparecen en el seguimiento a largo plazo. La pérdida del control esfinteriano nocturno, la necesidad de evacuar durante la noche y los episodios de ensuciamiento diurno son secuelas que producen un deterioro en la calidad de vida de los pacientes. ${ }^{9}$ Según algunos autores, las complicaciones sépticas ocurridas en la confección del reservorio son un potente predictor de la falla del reservorio. ${ }^{10}$ Por todo esto es importante documentar en detalle las complicaciones postoperatorias y su eventual impacto en los resultados funcionales a corto y largo plazo. ${ }^{11}$

El objetivo de este trabajo es dar a conocer la experiencia y resultados a corto plazo del uso de RIARA en "J" luego de proctocolectomía en el manejo de CUy PAF.

\section{METODOLOGÍA}

En este estudio se analiza los resultados inmediatos (complicaciones < 30 días) y seguimiento a largo plazo de todos los pacientes sometidos a una RIARA en "J" con técnica grapada entre 2014 y 2016 de los pacientes hospitalizados en la Clínica Guayaquil. Para la evaluación de los resultados funcionales se incluyeron los pacientes que tengan al menos un año de seguimiento luego del cierre de la ileostomía de protección y se excluyen a los que tengan menor a 1 año de colectomia, pacientes con trauma penetrante de abdomen que se resolvió con colectomías.

Se entiende como cirugía en tres tiempos: i) la realización de una colectomía total con ileostomía terminal y cierre del muñón rectal debido a una urgencia quirúrgica en el primer tiempo; ii) la RIARA protegida por una ileostomía en asa en el segundo tiempo; y iii) el cierre de la ileostomía en el tercero. La técnica ha sido descrita en detalle en el ámbito nacional y básicamente se realiza en todos los pacientes tanto el reservorio como la anastomosis reservorio-anal mediante suturas mecánicas.

Brevemente, con el paciente en posición de litotomía-Trendelemburg, se inicia el primer tiempo electivo de la panproctocolectomía, en la que se libera el colon abdominal, ligando por separado los vasos nutricios del colon y completando la liberación del recto pelviano. Se efectúa cierre del muñón con una carga de la grapadora lineal (TA o roticulador según disponibilidad) y refuerzo de sutura con puntos quirúrgicos alrededor de la sutura mecánica. Posterior a esto se realiza comprobación de su hermeticidad en forma digital. Se realiza sección del íleon a nivel de la válvula ileocecal conservando los vasos ileocólicos y se confecciona un reservorio en J de $15 \mathrm{~cm}$ de largo plegando el íleon terminal sobre sí mismo mediante 2 cargas consecutivas de lineal cortante de $80 \mathrm{~mm}$ que confeccionan la anastomosis latero-lateral (Figura 1).

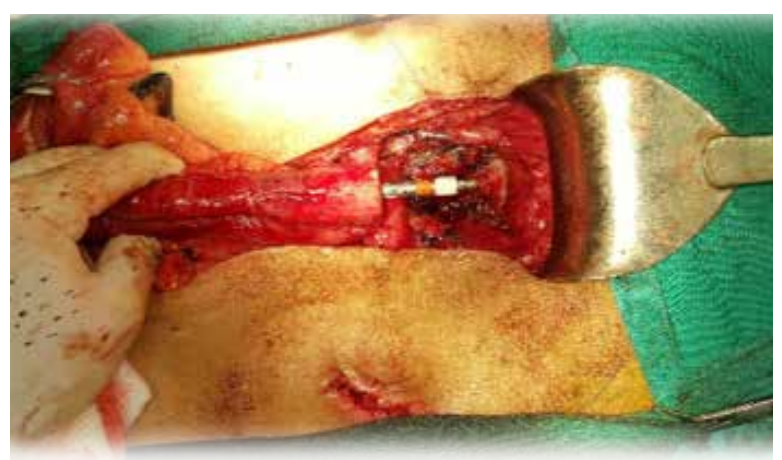

Figura 1. Anastomosis de las 2 porciones de íleon mediante GIA. 
Luego se efectúa el cierre del íleon terminal con una carga adicional de lineal cortante y un segundo plano invaginante con vicryl 3.0. Se hace una jareta en el extremo distal del reservorio que debe alcanzar el plano del pubis e instalación del cabezal del instrumento circular. Se anastomosa el reservorio al muñón rectal con técnica de doble grapado y comprobación de hermeticidad de ésta y el reservorio con insuflación de aire a través de una sonda Foley introducida por el ano(Figura 2).

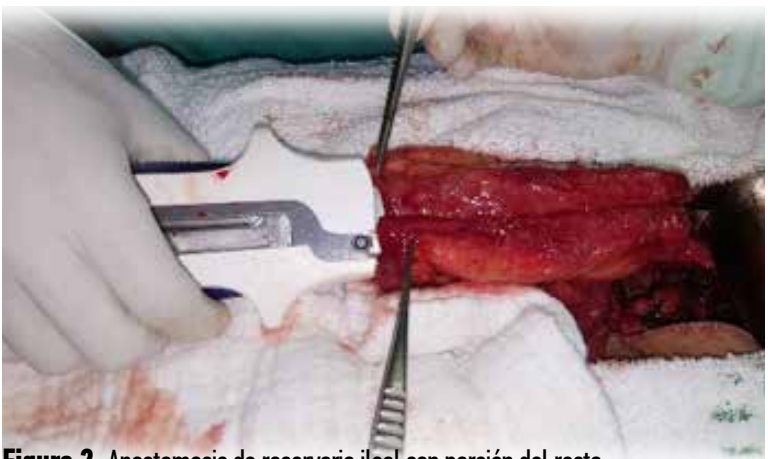

Figura 2. Anastomosis de reservorio ileal con porción del recto.

RESULTADOS

En el período señalado se realizaron 11 RIARA (8 hombres y 3 mujeres) en forma consecutiva con anastomosis mecánica (Figura 1).

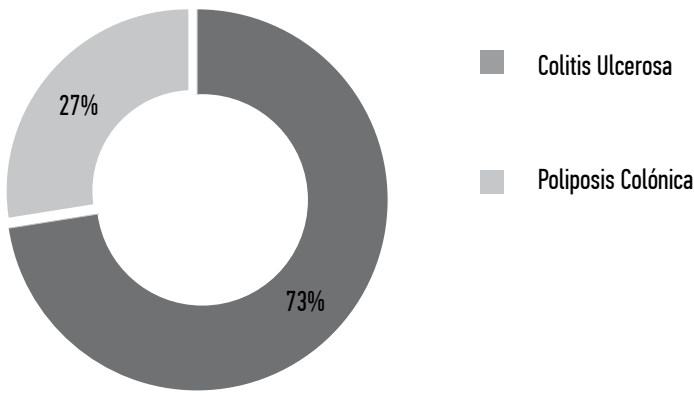

Figura 1. Distribución por patología a la que se efectuó el reservorio ileal.

Las indicaciones fueron CU grave en 8 casos (grupo CU) y poliposis colónica en 3 (grupo $\mathrm{PAF}$ ). En el grupo CU los 3 pacientes fueron operados en 2 tiempos. El cierre del muñón ano-rectal se realizó con roticulador de $30 \mathrm{~mm}$ en $80 \%$ de los casos y con un TA $30 \mathrm{~mm}$ en el resto. La anastomosis de la RIARA se efectuó con un circular de $28 \mathrm{~mm}$ en 6 casos, de $31 \mathrm{~mm}$ en 4 casos y de $25 \mathrm{~mm}$ en 1 , quedando a $2 \mathrm{~cm}$ del margen anal como promedio. La morbilidad global postoperatoria se eleva al 19\% en el grupo CU mientras que la morbilidad global en el grupo PAF fue $22 \%$ (Figura 2 ).

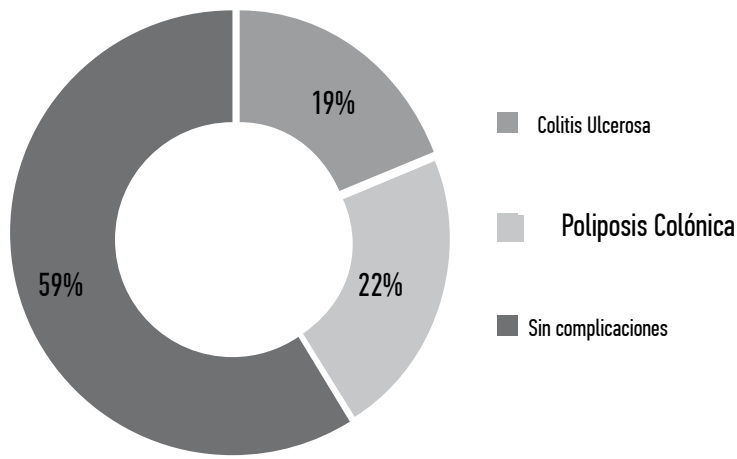

Figura 2. Morbilidad según patología luego efectuado el procedimiento.

No hubo diferencias significativas en los resultados funcionales de acuerdo a la etiología de base. El promedio de evacuaciones en 24 horas fue de 4 , sin diferencias observadas entre ambos grupos y dichos datos tomados 1 mes posterior a la alta médica del paciente. Posterior al alta el $70 \%$ de los pacientes tenían al menos una evacuación nocturna y el 33\% refería ensuciamiento nocturno. Sólo 2 pacientes llevan una dieta restrictiva y usan medicamentos como la Loperamida (Tabla 1).

TABLA 1. RESULTADOS FUNCIONALES SEGÚN SCORE DE ÖRESLAND

\begin{tabular}{|c|c|c|c|}
\hline & \multicolumn{2}{|c|}{ PUNTAJE } & N \\
\hline \multicolumn{4}{|l|}{ Evacuaciones } \\
\hline \multirow{3}{*}{ Día } & $\leq 4$ & 0 & 8 \\
\hline & 5 & 1 & 2 \\
\hline & $\geq 6$ & 2 & 1 \\
\hline \multirow{3}{*}{ Noche } & 0 & 0 & 1 \\
\hline & 1 & 1 & 7 \\
\hline & 2 & 2 & 3 \\
\hline Urgencia* & & 1 & 2 \\
\hline Disquezia** & & 1 & 1 \\
\hline \multicolumn{4}{|l|}{ Ensuciamiento } \\
\hline Día & $\begin{array}{r}> \\
1 / \text { semana }\end{array}$ & 1 & 2 \\
\hline Noche & $\begin{array}{r}> \\
1 / \text { semana }\end{array}$ & 1 & 3 \\
\hline \multicolumn{4}{|l|}{ Irritación perianal } \\
\hline Ocasional & & 1 & 1 \\
\hline Permanente & & 2 & 2 \\
\hline \multicolumn{4}{|l|}{ Uso de paño protector } \\
\hline Día & $\begin{array}{r}> \\
1 / \text { semana }\end{array}$ & 1 & 2 \\
\hline Noche & $\begin{array}{r}> \\
1 / \text { semana }\end{array}$ & 1 & 3 \\
\hline Restricción dietética & & 1 & 2 \\
\hline $\begin{array}{l}\text { Medicamentos } \\
\text { (Loperamida) }\end{array}$ & & 1 & 1 \\
\hline Alteración de vida social & & 1 & 2 \\
\hline
\end{tabular}




\section{DISCUSIÓN}

El manejo de la CU crónica inespecífica y la PAF requerirá cirugía en un tercio de los pacientes durante la historia natural de la enfermedad, siendo la indicación principal la falta de respuesta al tratamiento médico o el desarrollo de cáncer. ${ }^{12} \mathrm{De}$ las técnicas quirúrgicas actuales, la proctocolectomía restaurativa con RIARA en "J" ofrece mayores beneficios. A pesar de ello, la morbilidad de la técnica es muy elevada y los resultados a largo plazo dependen del seguimiento de los pacientes. ${ }^{13}$ Esto es cierto en países con poca experiencia justificada por la incidencia tan baja de la enfermedad. ${ }^{14} \mathrm{Las}$ series previamente publicadas demuestran las ventajas de este tratamiento quirúrgico que, además de resecar la mucosa enferma, disminuye la posibilidad de desarrollar una neoplasia colorrectal, permitiendo una función intestinal adecuada particularmente en términos de frecuencia de evacuaciones y continencia. ${ }^{15}$

Se conoce que la incidencia de CU crónica inespecífica es mayor en países industrializados como Estados Unidos de Norteamérica, Reino Unido, Noruega y Suecia, con una incidencia de 6 a 12 casos por 100 mil habitantes al año. En Panamá y Argentina es de 1.2 y 2.2 , respectivamente, por 100 mil habitantes al año. ${ }^{16}$

\section{CONCLUSIÓN}

De acuerdo a los resultados obtenidos se constató que la morbilidad global postoperatoria se elevó al $19 \%$ en el grupo CU presentando como causas más frecuentes cuadro broncopulmonar en 2 casos, drenaje seroso prolongado sin fiebre asociada en 1 caso y colección pélvica que requirió drenaje quirúrgico en 1 caso. La morbilidad global en el grupo PAF fue $22 \%$ siendo más frecuente síndrome febril sin colección demostrable en 1 caso y en otro caso colección pélvica que no requirió drenaje quirúrgico y con buena respuesta al tratamiento antibiótico empírico.

Se efectuó reservoriografía con bario en $60 \%$ de los casos previo a la reconstitución del tránsito, sin que se haya demostrado filtración o algún trayecto fistuloso. Aunque todos los pacientes reportan tener algún grado de alteración de la vida social, 9 de los pacientes estudiados dijeron ser capaces de tener una ocupación de tiempo completo. Los pacientes reportaron estar muy satisfechos con la intervención y la recomendarían plenamente. Los pacientes con una evaluación deficiente de su calidad de vida correspondían a 2 casos operados por CU.
Frente a la pregunta si aceptarían una ileostomía para mejorar su calidad de vida, los 2 pacientes con una evaluación deficiente rechazaron esta opción en forma categórica. Al comparar los resultados funcionales en diferentes etapas y en el seguimiento a largo plazo, se constata que luego de un año y medio desde el cierre de la ileostomía no había cambios significativos en las funciones evaluadas y la mayor parte de los pacientes tenían una función satisfactoria según la escala de Öresland. Altos porcentajes de satisfacción respecto a la función evacuatoria, también son obtenidos en centros con mayor número de cirugías (Tabla 2).

TABLA 2. RESULTADOS FUNCIONALES SEGÚN PATOLOGÍA DE BASE

\begin{tabular}{lrrr} 
PUNTAJE ÖRESLAND & $\begin{array}{r}\text { COLITIS } \\
\text { ULCEROSA }\end{array}$ & PAF & TOTAL (\%) \\
\hline $0-3$ satisfactorio & 6 & 2 & $8(72)$ \\
\hline $4-6$ aceptable & 1 & 1 & $2(18)$ \\
\hline 7 deficiente & $1^{*}$ & 0 & $1(9)$ \\
\hline Total & 8 & 2 & $11(100)$ \\
\hline
\end{tabular}

*Paciente sometido a limpieza quirúrgica y drenaje de absceso.

\section{REFERENCIAS BIBLIOGRÁFICAS}

1. Holubar S, Hyman. N. Continence alterations after ileal pouch-anal anastomosis do not diminish quality of life. Dis Colon Rectum. 2003: p. 46:1489-91..

2. Fazio V, Ziv Y, Church J, Oakley J, Lavery I, Milsom J, et al. Ileal pouch-anal anastomosis: complications and function in 1005 patients. Ann Surg. Ann Surg. 1995; p. 222:120-7. 2.

3. Sagap, Remzi, Hammel, Fazio.. Factors associated with failure in managing pelvic sepsis after ileal pouch-anal anastomosis (IPAA)-a multivariate analysis. Surgery. 2006; p. 140:691-703.

4. Berndtsson I, Lindholm E, Öresland T, Börjesson. L. Long-term outcome after ileal pouch-anal anastomosis: function and health-related quality of life. Dis Colon Rectum. 2007; p. 50:1545-52.

5. Jensen C, Vergara J, Pérez G. Alternativas quirúrgicas en la colitis ulcerosa idiopática. Rev Chil Cir. 1995; p. 47:230-4. 7.

6. Camilleri-Brennan J, Munro A, Steele. R. Does an ileoanal pouch offer a better quality of life than a permanent. Gastrointest Surg. 2003; p. 7:814-9.

7. Zúñiga , López, Rahmer, Quintana , Duarte , Donoso.. Reservorio ileal en el tratamiento quirúrgico de la colitis ulcerosa. Rev Chil Cir. 1997; p. 49:69-75. 6.

8. Öresland, Fasth, Nordgren ,. H. The clinical and functional outcome after restorative proctocolectomy. A prospective study in 100 patients. Int J Colorectal Dis. 1989; p. 4:50-6. 
9. Block M, Börjesson L, Lindholm E, Öresland. T. Pouch design and long-term functional outcome after ileal pouch-anal anastomosis. Br J Surg. 2009; p. 96:527-32.

10. Zárate, Zúñiga, Pinedo, López, Molina ,. V. Reservorio ileal con anastomosis reservorio anal por colitis ulcerosa: complicaciones y resultados a largo plazo. Rev Med Chile. 2008; p. 136:467-74. 15.

11. MICHELASSI , HURST.. Restorative proctocolectomy with J-pouch ileoanal anastomosis. Arch Surg. 2000; p. 135: 347-53.

12. BEMELMAN, DUNKER, SLORS, GOUMA.. Laparoscopic surgery for inflammatory bowel disease: current concepts. Scand J Gastroenterol. 2002; p. 236: $54-9$.
13. Forbes, O'Connor, Victor, Cohen, McLeod.. Sepsis is a major predictor of failure after ileal pouch-anal anastomosis. 52197581st ed.: Dis Colon Rectum; 2009.

14. PARKS , NICHOLS.. Proctocolectomy with preservation of the sphincter. Br Med J. 1978; p. 2: 85-8.

15. Tulchinsky, Cohen, Nicholls.. Salvage surgery after restorative proctocolectomy. Br J Surg. 2003; p. 90:909-921.

16. Mery C, Robles G. Colitis ulcerativa crónica inespecífica. I: Generalidades y diagnóstico. TakahashiMonroy T, ed. In: Colon, recto y ano. Enfermedades medico-quirúrgicas. México: Editores de Textos Mexicanos. 2003; p. 408-417. 